\title{
Support Function Representation for Curvature Dependent Surface Sampling
}

\author{
Maria Lucia Sampoli ${ }^{1}$, Bert Jüttler ${ }^{2}$ \\ ${ }^{1}$ Department of Mathematics and Computer Sciences, University of Siena, \\ Pian dei Mantellini 44, 53100 Siena, Italy \\ sampoli@unisi.it \\ ${ }^{2}$ Institute of Applied Geometry, Johannes Kepler University, \\ Altenberger Str. 69, 4040 Linz, Austria \\ Bert.Juettler@jku.at
}

\begin{abstract}
In many applications it is required to have a curvature-dependent surface sampling, based on a local shape analysis. In this work we show how this can be achieved by using the support function (SF) representation of a surface. This representation, a classical tool in Convex Geometry, has been recently considered in CAD problems for computing surface offsets and for analyzing curvatures. Starting from the observation that triangular Bézier spline surfaces have quite simple support functions, we approximate any given free-form surface by a quadratic triangular Bézier spline surface. Then the corresponding approximate SF representation can be efficiently exploited to produce a curvature dependent sampling of the approximated surface.
\end{abstract}

Keywords: Support function, triangular Bézier surfaces, quadratic patches, data sampling.

\section{Introduction}

One of the main tasks of Computer Aided Geometric Design is to represent curves and surfaces, satisfying some interpolations or approximation conditions, in a way which allows an easy manipulation for further applications (see for instance [6]). The most important performed operations are usually offsetting, convolution computations, feature lines computations and extraction of information on curvatures and other geometric quantities.

Among all representations, NURBS (Non Uniform Rational B-Spline) are widely used and therefore a big deal of research has been done, for example, in order to detect subsets of NURBS closed under offsetting or the more general convolution operation, or to characterize specific tools for defining the geometric features of the curves and surfaces. 
In this context quite recently it was noted that a simple approach to deal with offsets and convolutions is to use the support function (SF) representation of surfaces. The support function representation is a classical tool in the field of Convex Geometry (see e.g. [3], [8]); it consists in describing a surface by the distance of its tangent planes to the origin of the coordinate system, and such a distance is seen as a function on the unit sphere. The surface can be then recovered from its support function by computing the envelope of the tangent planes.

The application of support function representation to problems from Computer Aided Design was noted for the first time in [14], but only recently has been investigated effectively, $[9,16,17]$. In these papers the shapes (curves and surfaces) which can be described by particular types of support functions -polynomial, rational, or piecewise linear- are considered and their geometric properties are discussed. In particular it is shown that the class of curves and surfaces with (piecewise) polynomial support functions is closed under convolutions, offsetting, rotations and translations. Indeed these operations correspond to simple algebraic operations of the corresponding support functions. Moreover we can see that the SF representation leads also to particularly simple expressions for quantities and mappings governing the differential geometry of the surfaces.

In this paper the approximation of a free-form surface with a quadratic triangular Bézier spline is considered. Such an approximation is done, following [7], by considering a $C^{1}$ quadratic spline quasi-interpolant. This implies the approximation of the corresponding support function. We see that each quadratic Bézier triangular patch has support function given by a rational function defined over a spherical triangle whose boundaries are conic sections. Therefore the support function of a triangular Bézier spline is given by a, possibly multi-valued, rational function defined over a partition of spherical triangles on the unit sphere.

The support function of a free-form surface is then approximated by the support function of the corresponding triangular Bézier spline approximation. In this way we can exploit this last SF representation to extract geometric information of the surface and to manipulate it for further modelling.

As an interesting application we show how to determine a curvature dependent surface sampling. The key idea is given by observing that a uniform point set on the unit sphere can be mapped through the envelope operator (defined from the SF) to a curvature dependent point set on the surface.

The remainder of the paper is divided into four sections. In the next section we review the main definitions and properties of support function representation of a surface. In Sect. 3 the approximation of general surfaces 
by quadratic ones is studied and the computation of the corresponding piecewise support functions is considered. Then Sect. 4 will be devoted to the computation of curvature-adaptive sampling points: the method is first presented in the univariate case and then the surface case is considered. Section 5 concludes the paper.

\section{Preliminaries}

Given a surface $\mathbf{x}(u, v): \Omega \rightarrow \mathbb{R}^{3}:(u, v) \mapsto \mathbf{x}(u, v)$, each point has an associated unit normal

$$
\mathbf{N}: \Omega \rightarrow \mathbb{S}^{2}:(u, v) \mapsto \mathbf{N}(u, v),
$$

where $\mathbb{S}^{2}$ is the unit sphere. The mapping $\mathbf{N}$, which depends smoothly on $u, v$ and defines an orientation of the surface, is bijective provided that the surface does not have parabolic or singular points.

Let us consider now the distance between the tangent plane and the origin

$$
H: \Omega \rightarrow \mathbb{R}:(u, v) \mapsto \mathbf{N}(u, v) \cdot \mathbf{x}(u, v) .
$$

Then the support function is defined as the composition of the inverse map of $\mathbf{N}$ with the above function $H$

$$
h: \mathbb{S}^{2} \rightarrow \mathbb{R}: h=(\mathbf{N})^{-1} \circ H .
$$

The support function assigns to each unit normal the distance between the corresponding tangent plane and the origin of the coordinate system.

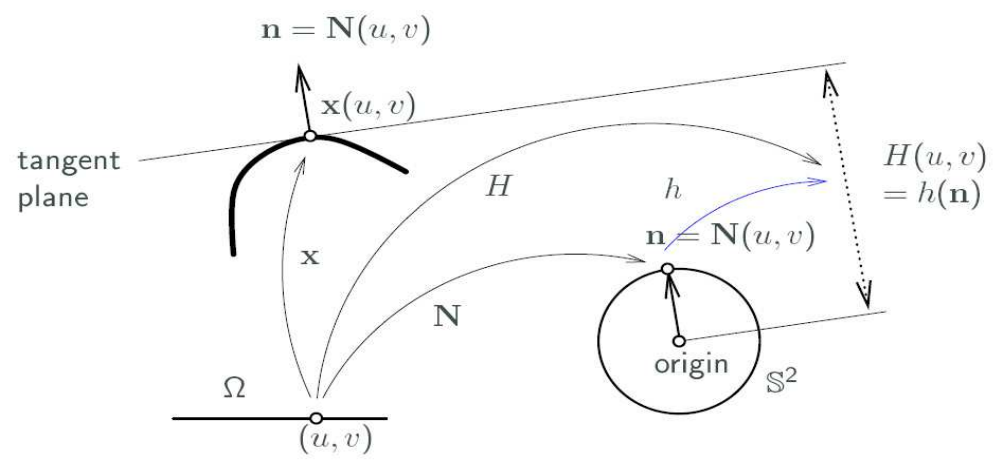

Fig. 1. A graphical scheme of the definition of the support function.

If a support function $h: D \rightarrow \mathbb{R}$ is given, where $D \subseteq \mathbb{S}^{2}$, then the associated surface is obtained by computing the envelope of the tangent 
planes

$$
\{\mathbf{p}: h(\mathbf{n})=\mathbf{n} \cdot \mathbf{p}\}, \mathbf{n} \in D .
$$

More precisely, for any $\mathbf{n} \in D$ we can compute the point on the envelope surface as

$$
\mathbf{x}_{h}: \mathbf{n} \mapsto \mathbf{x}_{h}(\mathbf{n})=h(\mathbf{n}) \mathbf{n}+\left(\nabla_{\mathbb{S}^{2}} h\right)(\mathbf{n}),
$$

where with $\nabla_{\mathbb{S}^{2}}$ we indicate the embedded intrinsic gradient with respect to the unit sphere. If $h^{*}$ is the extension of $h$ defined over all $\mathbb{R}^{3}$ then the intrinsic gradient can be obtained by projecting the usual gradient into the tangent plane of the sphere,

$$
\left(\nabla_{\mathbb{S}^{2}} h\right)(\mathbf{n})=\left(\nabla h^{*}\right)(\mathbf{n})-\left[\left(\nabla h^{*}\right)(\mathbf{n}) \cdot \mathbf{n}\right] \mathbf{n} .
$$

In conclusion we can define the envelope operator $\mathcal{E}$ which associates a surface $\mathbf{x}_{h}$ to a support function $h$,

$$
\mathcal{E}: C^{1}\left(\mathbb{S}^{2}, \mathbb{R}\right) \rightarrow C\left(\mathbb{S}^{2}, \mathbb{R}^{3}\right): h \mapsto \mathbf{x}_{h} .
$$

We may note that the envelope operator $\mathcal{E}$ is a linear mapping and defines an isomorphism between the linear spaces $C^{1}(D, \mathbb{R})$ and its images, where the addition in the image space is given by the so called convolution of the surfaces (for more details see $[15,9]$ ).

\section{Approximation of surfaces and their support functions}

In general, given a quadratic surface patch

$$
\mathbf{p}(u, v)=\frac{1}{2} a_{20} u^{2}+a_{11} u v+\frac{1}{2} a_{02} v^{2}+a_{10} u+a_{01} v+a_{00},
$$

its support function can be found by eliminating $u, v$ from the following system of equations:

$$
\begin{gathered}
h(\mathbf{n})=\mathbf{n} \cdot \mathbf{p}(u, v), \\
\mathbf{n} \cdot \mathbf{p}_{u}(u, v)=0, \quad \mathbf{n} \cdot \mathbf{p}_{v}(u, v)=0
\end{gathered}
$$

The last two equations are linear in $u, v$, then we can make these parameters explicit with respect to $\mathbf{n}=\left(n_{1}, n_{2}, n_{3}\right)$ and substitute the result into in the first equation, finding the expression of the support function of the surface. By straightforward computation it is easy to see that we obtain a rational expression for $h$, which is given as the quotient of a cubic and a quadratic homogeneous polynomial. 
In particular our attention will be focused on quadratic triangular Bézier surface patches

(10)

$$
\mathbf{x}(u, v, w)=\sum_{i+j+k=2} B_{i, j, k}^{2}(u, v, w) \mathbf{b}_{i, j, k}, \quad u, v, w \geq 0, \quad u+v+w=1 .
$$

where $(u, v, w)$ are barycentric coordinates with respect to some domain triangle $\Delta \subset \mathbb{R}^{2}$, the basis functions $\frac{2}{i ! j ! k !} u^{i} v^{j} w^{k}$ are the bivariate Bernstein polynomials of degree 2 and the coefficient vectors $\mathbf{b}_{i, j, k}$ are called control points. This representation is very common in CAD applications for its simplicity and many useful geometric properties, for instance the surface patch is contained in the convex hull defined by the control points. An example is given in Fig. 2. For more details see for instance [6].

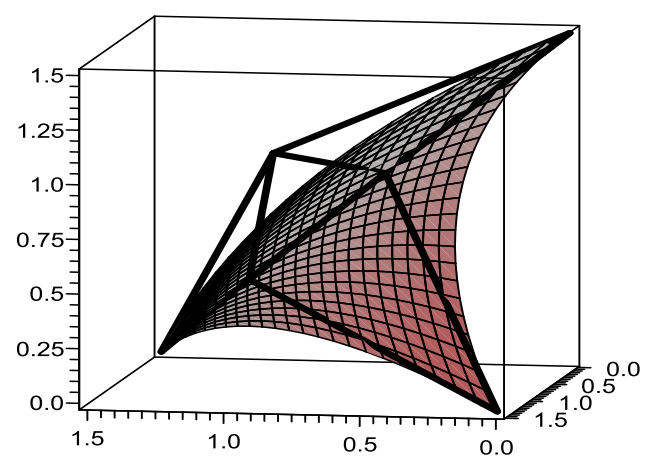

Fig. 2. An example of a quadratic triangular Bézier patch.

Recently it was found out that quadratic triangular Bézier splines also belong to the class of surfaces with odd rational support functions and therefore it can be proved that they belong to the family of surfaces which can be equipped with a linear field of normal vectors. This nice feature allows the exact computation of a rational parameterization of offsets and convolution surfaces, $[10,11,12,15]$. A Bézier patch, by construction, is uniquely defined once its control points are assigned. A triangular quadratic patch is defined by 6 control points: the three vertices of the triangle and one additional point for each side (we have no inner control points in the quadratic case). Considering the patch of Fig. 2, its control points are given by

$$
\begin{aligned}
\mathbf{b}_{2,0,0} & =(2,0,0)^{T}, \mathbf{b}_{0,2,0}=(0,2,0)^{T}, \mathbf{b}_{0,0,2}=(0,0,2)^{T}, \\
\mathbf{b}_{1,1,0} & =\left(\frac{3}{2}, 0,0\right)^{T}, \mathbf{b}_{1,0,1}=\left(0, \frac{3}{2}, 0\right)^{T}, \mathbf{b}_{0,1,1}=\left(0,0, \frac{3}{2}\right)^{T},
\end{aligned}
$$


then the expression of the support function is given by

$h(\mathbf{n})=\frac{5\left(n_{1}^{3}+n_{2}^{3}+n_{3}^{3}\right)+4\left(n_{1}^{2} n_{2}+n_{1}^{2} n_{3}+n_{1} n_{2}^{2}+n_{1} n_{3}^{2}+n_{3} n_{2}^{2}+n_{3}^{2} n_{2}\right)+13 n_{1} n_{2} n_{3}}{2\left(n_{1}^{2}+3 n_{1} n_{2}+n_{2}^{2}+3 n_{1} n_{3}+3 n_{2} n_{3}+n_{3}^{2}\right)}$.

In the next paragraph we present a method to approximate general free-form surfaces by quadratic triangular Bézier splines.

\subsection{Quadratic spline approximation}

Given a free-form parametric surface we want to build a quadratic triangular Bézier spline of the form (10), which approximates it.

The construction is done component by component. For each component we may consider the $C^{1}$ quadratic spline quasi-interpolant described in $[4,7]$. For the sake of completeness we report briefly the basic steps of the construction.

In the parameter domain $\Omega$, that without loss of generality can be assumed equal to $[0,1]^{2}$, we consider a rectangular grid given by a set of $m n$ isoparametric lines and then we endow it with the so-called crisscross triangulation constructed taking all the diagonals of each subrectangle $\Omega_{i, j}=\left[\frac{i}{n}, \frac{i+1}{n}\right] \times\left[\frac{j}{m} \frac{j+1}{m}\right], i=0 \ldots n-1, j=0, \ldots, m-1$.

Given a function $f$ defined in $\Omega$, we then consider the $C^{1}$ quadratic spline quasi-interpolant defined by

$$
Q f=\sum_{i=0}^{n} \sum_{j=0}^{m} \mu_{i j}(f) \mathcal{B}_{i j}
$$

where $\mathcal{B}_{i j}$ are the classical $C^{1}$ quadratic box splines obtained as translates of the Zwart-Powell element (see [5]), and $\mu_{i j}(f)$ are appropriate linear combinations of vertex values and/or centre values of adjacent subrectangles $([4,7])$. We note that various choices for the coefficients $\mu_{i j}(f)$ are possible, giving rise to different quasi-interpolants, sharing optimal properties.

We can see that these quasi-interpolant operators reproduce exactly the space of bivariate polynomials and produce optimal approximation order for smooth functions and their derivatives. More precisely, denoting with $k=\max \left\{\frac{1}{n}, \frac{1}{m}\right\}$, with $\|\cdot\|_{\infty, \Omega}=\|\cdot\|_{\infty}=$ the supremum norm over $\Omega$, and with $D^{\beta}=\frac{\partial^{|\beta|}}{\partial u^{\beta_{1}} \partial v^{\beta_{2}}},|\beta|=\beta_{1}+\beta_{2}$, we have $([4,7])$

$$
\|f-Q f\|_{\infty} \leq C_{0} k^{3}, \quad\left\|D^{\beta}(f-Q f)\right\|_{\infty} \leq C_{1} k^{2} \text { with }|\beta|=1 .
$$

where the constants $C_{i}$ depends solely on the function $\mathrm{f}$.

In the case of parametric surfaces, we can obtain the above bounds for each component and taking the maximum norm we can conclude that the 
maximum difference between a surface $\mathbf{x}(u, v)$ and its approximant $\mathbf{p}(u, v)$ can be bounded by $\tilde{C} k^{3}$.

\subsection{Support function approximation}

Given a quadratic triangular Bézier spline, let us study now its support function.

Let us consider first a single triangular patch. In order to construct its support function we have to determine the region on the unit sphere which is the domain of the support function, that is the image of the mapping $N$ introduced in (1). We should assume that the Bézier triangle does not contain any parabolic points. In [1] it was noted that the parabolic points of quadratic Bézier triangles determine curves which are images of straight lines in the parameter domain. Therefore in these cases it is sufficient to split the triangle along these lines, in order to exclude parabolic points.

Now, considering the points on the surface, the domain of the support function can be also seen as the image of the Gaussian map of the patch.

$$
\mathbf{G}: M \in \mathbb{R}^{3} \rightarrow \mathbb{S}^{2}: \mathbf{p} \mapsto \mathbf{n}_{\mathbf{p}}
$$

With the help of a computer algebra system it is easy to see that the Gaussian image of a quadratic triangular patch is a spherical triangle with curved boundaries (conic sections). Such curves are obtained by the intersection of the sphere with three quadratic cones. If one of these cones is singular the spherical triangle may degenerate into a biangle. The support function will
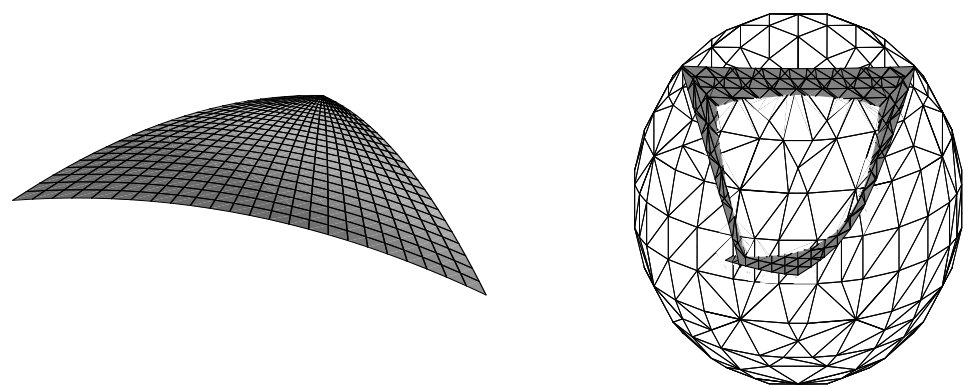

Fig. 3. A quadratic triangular patch and the domain of its support function on the unit sphere.

be then given eliminating $u, v$ in (9) and solving them with respect to $\mathbf{n}$.

In the case of a quadratic spline the support function will be obtained collecting the support functions of all triangular patches constituting the spline. The resulting support function will be given by a, possibly multivalued, piecewise $C^{0}$ rational function on the unit sphere, defined over a 
collection of curved spherical triangles.

Remark 3.1. As we have said in the previous section, in order to have a well defined support function, we have to exclude singular points and parabolic ones. The last case can be checked considering for each triangular patch the sign of the principal curvatures. In particular we check the sign of the Gaussian curvature (achieved by computing the first and the second fundamental forms) and we classify the patches in elliptic and hyperbolic ones according to their curvature sign. Then we can treat them separately. The patches with some points of zero curvature can be subdivided till small (up to a fixed tolerance) regions containing isolated points or curves of parabolic points are detected According to the various applications these region will be handled differently.

Regarding the approximation order, from the error estimates (13), we can prove that the distance between the SF of a given surface $h_{x}$ and that of its approximant $h_{p}$ can be bounded by $\hat{C} k^{3}$.

\section{Curvature-dependent sampling}

In Computer Aided Geometric Design (CAGD), curves and surfaces have two standard representations: parametric and implicit. The parametric representation offers a number of advantages, e.g., simple techniques for display and for analyzing the geometric properties as well as fast generation of point meshes, fast visualization and interactive modelling. On the other hand, implicitly defined surfaces are better suited in many applications, for instance for the possibility of defining solids. Indeed the representation of geometric objects based on volumetric data structures guarantee e.g., fast surface interrogation or Boolean operations such as intersection and union. However, surface based algorithms like shape optimization (fairing) or freeform modelling often need a topological manifold representation where neighborhood information within the surface is explicitly available. Consequently, it is necessary to find effective conversion algorithms to generate explicit surface descriptions for the geometry which is implicitly defined by a volumetric data set. Since volume data is usually sampled on a regular grid with a given step width, we often observe severe alias artifacts at sharp features on the extracted surfaces. Then it is crucial to have a surface sampling which is feature sensitive and thus reduces these alias effects.

Surface sampling is used in many other applications such as Computer Graphics and Visualization (e.g.in biomedical problems), as well as subdivision and surface reconstruction (see for instance $[2,13]$ ).

The aim of this section is to present a simple method to determine a 
surface sampling which is based on a local shape analysis. The main tool to achieve this is to use the support function representation of a surface.

\subsection{The curve case}

It is convenient to illustrate to idea first in the curve case. Given a quadratic spline it is always defined the corresponding support function. In this case the domain will be the unit circle. If we take a uniform set of points in the unit circle and we map back through the envelope operator defined by (7), we obtain a set of sampling points of the curve which is curvature dependent. This is basically due to the fact that the support function, being
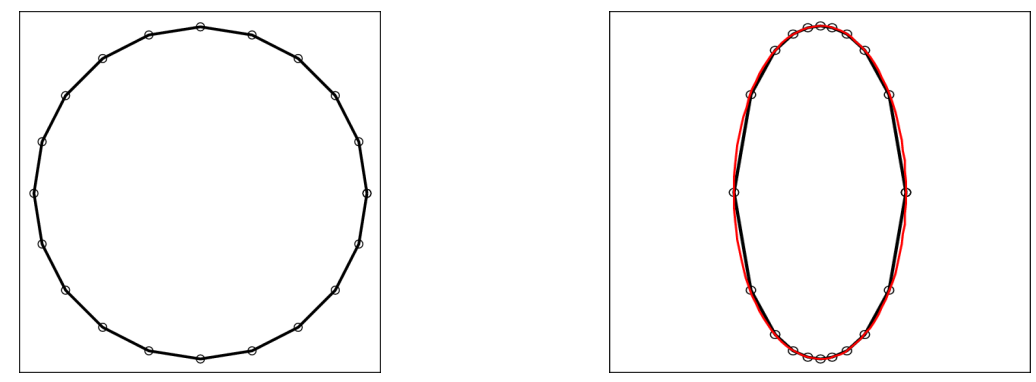

Fig. 4. A uniform point set on the unit circle is mapped in a curvature-dependent sampling on the curve.

defined in the Gaussian circle, by construction contains information about the curvature, see Fig. 4-5.
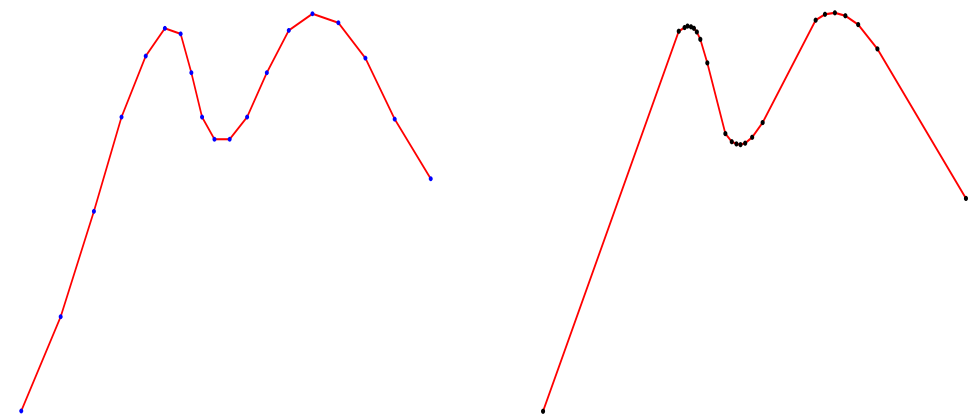

Fig. 5. Curve sampling. Left: the polygonal line connecting a uniformly sampled set of points in the curve. Right: the polygonal line connecting the curvature-dependent set.

The idea of sampling planar curves using an approach which takes into 
account curvature information was first investigated in [18], where the magnitude of the curvature signature function was studied.

\subsection{The surface case}

In the surface case we start from a uniformly distributed set of points into the unit sphere, for instance we can take the points coming from a uniform refinement of an icosahedron. In more detail having the quadratic triangular spline approximating a given surface, as we have seen in Sect. 3, the SF is given by a piecewise function defined over a partition of the unit sphere. Taking a point on the unit sphere if it belongs to the domain of the $\mathrm{SF}$, it will be mapped by the envelope operator into a point of the surface. The number of sampling points depends by the number of points on the unit sphere. Figures 6-7 show two examples, where in the first one two sets of sampling points are constructed.
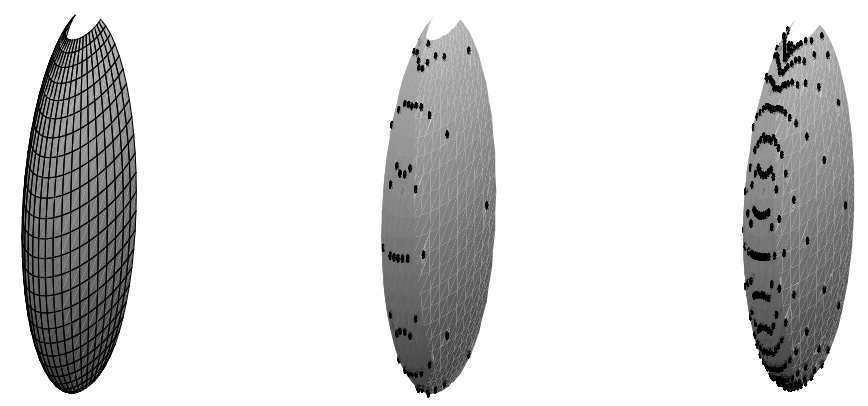

Fig. 6. Example of a part of an ellipsoid surface. Centre: a resulting sampling set. Right: a finer sampling.
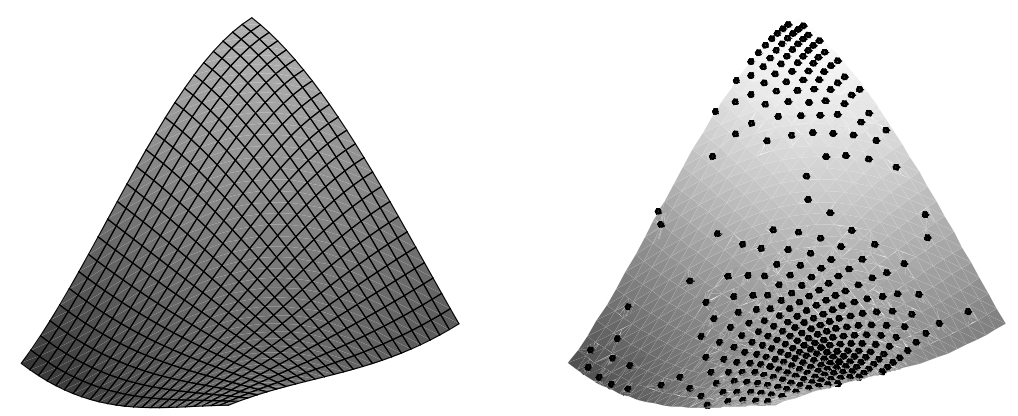

Fig. 7. Example of a non convex surface. Right: a resulting sampling set along with the approximating surface. 


\section{Conclusion}

In this paper we have shown how the support function representation can be used effectively to construct a sampling set of a surface which is curvature dependent and therefore preserves its main features.

In general the SF may be not explicitly available for generic surfaces and therefore, as an intermediate step, a method to efficiently approximate a given surface with a triangular Bézier spline is presented. In this way we can use the SF of quadratic triangular splines which can be explicitly computed.

\section{REFERENCES}

1. B. Bastl, B. Jüttler, J. Kosinka and M. Lávička, Computing exact rational offsets of quadratic triangular Bezier surface patches, Comp. Aided Design, 40 (2008), pp. 197-209.

2. J. D. Boissonnat and S. Oudot, Provably good surface sampling and approximation, ACM Intern. Conf. Proc. Series 23 (Eurographics 2003)(2003), pp. 9-18.

3. T. Bonnesen and W. Fenchel, Theory of Convex Bodies, BCS Associates, Moscow, Idaho, 1987.

4. C. Dagnino, P. Sablonnière, Error analysis for quadratic spline quasiinterpolants on non uniform criss-cross triangulations, Prépublication IRMAR 06-06, Rennes 2006.

5. C. de Boor, K. Höllig and S. Riemenschneider,Box Splines, SpringerVerlag, 1993.

6. G. Farin, J. Hoschek and M.-S. Kim editors, Hanbook of Computer Aided Geometric Design, Elsevier Science, Amsterdam, The Netherlands, 2002.

7. F. Foucher and P. Sablonnière, Approximating partial derivatives of first and second order by quadratic spline quasi-interpolants on uniform meshes, Math. and Comput. Simulation 77 (2008),pp. 202-208.

8. R. Gardner, Geometric Tomography, Cambridge University Press, Cambridge, 1995.

9. J. Gravesen, B. Jüttler and Z. Š́r, On rationally supported surfaces. Comp. Aided Geom. Design 25 (2008), pp. 320-331.

10. B. Jüttler and M. L. Sampoli, Hermite interpolation by piecewise polynomial surfaces with rational offsets, Comp. Aided Geom. Design $\mathbf{1 7}$ (2000), pp. 361-385. 
11. M. Lávička and B. Bastl, Rational hypersurfaces with rational convolutions, Comp. Aided Geom. Design, 24 (2007), pp. 410-426.

12. M. Peternell and B. Odehnal, Convolution surfaces of quadratic triangular Bézier surfaces, Comp. Aided Geom. Design, 25 (2008), pp. 116-129.

13. H. Pottmann, T. Steiner, M. Hofer, C. Haider, and A. Hanbury, The isophotic metric and its application to feature Ssensitive morphology on surfaces. In T. Pajdla and J. Matas editors, Computer Vision - ECCV 2004, Part IV, Lecture Notes in Computer Science vol. 3024 (2004), $560-572$.

14. M. Sabin, A class of surfaces closed under five important geometric operations, Technical Report No. VTO/MS/207, British Aircraft Corp. 1974.

15. M. L. Sampoli, M. Peternell and B. Jüttler, Rational surfaces with linear normals and their convolution with rational surfaces, Comp. Aided Geom. Design, 23 (2006), pp. 179-192.

16. Z. Š́r, J. Gravesen and B. Jüttler. Computing convolution and Minkowski sums via support function representation. In P. Chenin, T. Lyche and L. L. Schumaker, editors, Curve and Surface Design: Avignon 2006, pp. 244-253. Nashboro Press, Nashboro MN, 2007.

17. Z. Š́r, J. Gravesen and B. Jüttler, Curves and surfaces represented by polynomial support functions, Theoretical Comp. Science, 392(1-3) (2008), pp. 141-157.

18. T. Surazhsky and V. Surazhsky. Sampling planar curves using curvature-based shape analysis. In M. Dæhlen, K. Mørken and L. L. Schumaker, editors, Mathematical methods for curves and surfaces: Tromsø 2004, pp. 339-350. Nashboro Press, Nashboro MN, 2005. 